Article

\title{
Adaptive Weibull Multiplicative Model and Multilayer Perceptron Neural Networks for Dark-Spot Detection from SAR Imagery
}

\author{
Alireza Taravat * and Natascha Oppelt \\ Remote Sensing \& Environmental Modelling Lab, Kiel University, Kiel 24098, Germany; \\ E-Mail: oppelt@geographie.uni-kiel.de \\ * Author to whom correspondence should be addressed; E-Mail: art23130@gmail.com; \\ Tel.: +49-431-880-3330.
}

External Editor: Ki-Hyun Kim

Received: 15 October 2014; in revised form: 18 November 2014 / Accepted: 25 November 2014 / Published: 2 December 2014

\begin{abstract}
Oil spills represent a major threat to ocean ecosystems and their environmental status. Previous studies have shown that Synthetic Aperture Radar (SAR), as its recording is independent of clouds and weather, can be effectively used for the detection and classification of oil spills. Dark formation detection is the first and critical stage in oil-spill detection procedures. In this paper, a novel approach for automated dark-spot detection in SAR imagery is presented. A new approach from the combination of adaptive Weibull Multiplicative Model (WMM) and MultiLayer Perceptron (MLP) neural networks is proposed to differentiate between dark spots and the background. The results have been compared with the results of a model combining non-adaptive WMM and pulse coupled neural networks. The presented approach overcomes the non-adaptive WMM filter setting parameters by developing an adaptive WMM model which is a step ahead towards a full automatic dark spot detection. The proposed approach was tested on 60 ENVISAT and ERS2 images which contained dark spots. For the overall dataset, an average accuracy of $94.65 \%$ was obtained. Our experimental results demonstrate that the proposed approach is very robust and effective where the non-adaptive WMM \& pulse coupled neural network (PCNN) model generates poor accuracies.
\end{abstract}

Keywords: segmentation; neural networks; dark spot detection; Synthetic Aperture Radar (SAR) 


\section{Introduction}

Remote sensing is a critical element for an effective response to marine oil spills and it is useful in several modes of oil spill control and detection, including large area surveillance, site specific monitoring and tactical assistance in emergencies [1]. Among all remote sensing techniques, the ability of Synthetic Aperture Radar (SAR) imaging to detect features on the ocean's surface makes this technology a powerful tool for monitoring oil spills [2-4]. Since SAR is an active sensor using microwave bands (L-Band (1-2 GHz), C-Band (4-8 GHz), X-Band (8-12 GHz)), it has day/night imaging capability and the ability to penetrate cloud cover [5-7].

On the ocean, the main backscattering process is surface scattering. The elementary scatterers are the waves whose wavelength satisfies the Bragg resonance condition, LBragg $=\lambda /(2 \sin \theta i)$, where LBragg is the wavelength of small-scale waves, $\lambda$ is the radar wavelength and $\theta \mathrm{i}$ is the incidence angle [8].

The ocean surface behaves as a mirror without Bragg waves, and most of the incident signal is reflected away in the specular direction. Oil spills damp small-scale Bragg waves and reduce the friction velocity more than oil-free surface because of their larger surface tension than water [8]; in a SAR image they therefore appear as regions with less brightness.

High frequency bands ( $\mathrm{X} \& \mathrm{C}$ band) are mainly used for oil spill detection because the damping effect is larger for the Bragg waves of shorter wavelengths. However, the use of L-band for extracting the dark features has already been demonstrated using ALOS-PALSAR and UAVSAR sensors [8-10].

Another factor influencing the backscattering behavior of the ocean surface is the wind speed. The wind speeds under which the dark features of oil spill can be distinguished from surrounding waters are approximately $3-14 \mathrm{~m} / \mathrm{s}$ [11]. Wind speeds of less than $\sim 3 \mathrm{~m} / \mathrm{s}$ smoothens equally the surfaces with and without oil spill; wind speeds higher than $\sim 14 \mathrm{~m} / \mathrm{s}$ churn the sea and roughen the surface resulting in a dumping effect which becomes negligible [8].

Oil dispersion also affects the visibility of oil spill in SAR images which is caused by dissolution, oxidization, and biodegradation. Thus, with increasing time from the oil discharge and with increasing wind speed, oil slicks become undetectable [8].

In general, oil spill detection is performed in three steps: dark spot detection (both oil spills and look-alikes), feature extraction and classification (necessary for discriminating oil spills from look-alikes which are ice, internal waves, natural organics, algae, and rain cells) [6,7,12-15]. The literature describes many efforts to develop automated and semi-automated systems for oil spill detection based on this procedure $[16,17]$. This study focuses on accurate dark-spot detection which is the most computationally intensive phase of the algorithm and is a critical step prior to feature information extraction and classification $[18,19]$.

A variety of algorithms for oil spill segmentation have been developed using different methodologies such as thresholding [15,20], support vector machines [21], and neural networks [22,23]. The approach presented in this paper is a combination of adaptive Weibull Multiplicative Model (WMM) and MultiLayer Perceptron (MLP) neural networks. The results will be compared with the results of Taravat, et al., which is a model from the combination of WMM and pulse coupled neural network (PCNN) techniques to differentiate between dark spots and background signals [3]. The reason for comparing the presented results with the non-adaptive WMM \& PCNN results is to test the capability of the adaptive WMM \& MLP 
model for increasing the accuracy of full automatic dark spot detection by using the non-adaptive WMM \& PCNN model.

In pulsed coupled neural networks model the setting of parameters represents the fundamental but complex task. Although it has already been proofed that pulsed coupled neural networks is very fast and accurate in dark spot detection, it still generates poor accuracies in some cases (not well-defined linear dark spots and not well-defined massive dark spots) [3].

The presented approach overcomes the non-adaptive WMM filter setting parameters by developing an adaptive WMM model. Furthermore a pixel based MLP neural network has been applied to check the capability of a pixel based classification in order to increase the accuracy of the model discussed in Taravat et al. [3].

\section{Methods}

The first step of dark feature detection is applying a filter [11,20]. Liu et al. used a $3 \times 3$ Lee filter, followed by a $5 \times 5$ Lee filter and a $7 \times 7$ Median filter applied to the original image [24]. Topouzelis et al . used a combination of the Lee and Local Region filters [22]. The combination applied to his study includes application of a $3 \times 3$ Lee filter to the original image, followed by a $5 \times 5$ Lee filter and a $7 \times 7$ Local Region filter [13]. In all of these kind of filters, it has been assumed that the real and the imaginary parts of the received wave follow Gaussian distribution which is in turn lead to Rayleigh distribution [25]. Another popular filter is the Weibull Multiplicative Filter which has shown high degree of success in modeling sea clutter $[3,26]$.

\subsection{Adaptive Weibull Multiplicative Filter (WMM)}

In this study WMM (with the assumption that the amplitude or the intensity image has the Weibull distribution [3]) is used in order to remove speckle and to enhance the contrast between the dark spot and the background $[3,26]$. The extraction of the texture image from the Weibull-distributed SAR image employs the local estimation of the scale and form parameters of the Weibull distribution [3,26];

The Weibull-distributed random variable $x$ with form parameter $\gamma_{x}>0$ and scale parameter $\beta_{x}>0$, has a probability density function given by:

$$
f(x)=\frac{\gamma_{x}}{\beta_{x}}\left(\frac{x}{\beta_{x}}\right)^{\gamma_{x}-1} \exp \left[-\left(\frac{x}{\beta_{x}}\right)^{\gamma_{x}}\right]
$$

The m-order moment can be expressed as:

$$
E\left[x^{m}\right]=m \beta_{x}^{m} \Gamma\left(\mathrm{m} / \gamma_{x}\right) / \gamma_{x}
$$

For $\gamma_{x}=2$, the Weibull distribution becomes a Rayleigh distribution, for $\gamma_{x}=1$, it becomes an exponential distribution. It can be shown that $x^{a}$ with $a>0$ is also Weibull distributed. If $z=x^{a}(z)$ with form and scale parameters given by, $\gamma_{z}=\gamma_{x} / a$ and $\beta_{z}=\beta_{x}^{a}$ follows that:

$$
f(z)=\frac{\gamma_{z}}{\beta_{z}}\left(\frac{z}{\beta_{z}}\right)^{\gamma_{z}-1} \exp \left[-\left(\frac{z}{\beta_{z}}\right)^{\gamma_{z}}\right]
$$

Consider $b$, with $a>b>0$ in such a way that: 


$$
\mathrm{z}=x^{a}=x^{b} x^{a-b}=\frac{x^{b}}{E\left[x^{b}\right]} E\left[x^{b}\right] x^{a-b}=s t
$$

where $s$ is the speckle, with unitary mean and $t$ is the texture of the Weibull-distributed variable $z . z$ is the variable for the SAR image.

$$
\mathrm{s}=x^{b} / E\left[x^{b}\right] \mathrm{t}=x^{a-b} E\left[x^{b}\right]
$$

In this form, it is possible to express $z$ as a multiplication of $s$ by $t$, where $\mathrm{s}$ is the speckle and $t$ is the texture of the Weibull-distributed variable $z$. The texture $t$ has Weibull distribution with form and scale parameter given, respectively, by:

$$
\gamma_{t}=\gamma_{x} /(a-b) \beta_{t}=\beta_{x}^{a-b} E\left[x^{b}\right]
$$

and the speckle has Weibull distribution with form and scale parameter given, respectively, by:

$$
\gamma_{s}=\gamma_{x} / b \beta_{s}=\beta_{x}^{b} / E\left[x^{b}\right]
$$

Let $p=b / a, 0 \leq p<1$ Then

$$
\mathrm{t}=x^{a-b} E\left[x^{b}\right]=x^{a(p-1)} E\left[x^{a p}\right]=z^{(1-p)} E\left[z^{p}\right]
$$

using p-order moment equation $\mathrm{E}\left[z^{p}\right]$ :

$$
t=p \beta_{z}^{p} \Gamma\left(\mathrm{p} / \gamma_{z}\right) \mathrm{z}^{1-p} / \gamma_{z}
$$

where $t$ can be considered as the filtered image and the factor $0 \leq p<1$ (which is set manually in non-adaptive WMM) gives the filtering intensity (Figure 1). In adaptive WMM model, the form parameter $\gamma_{s}$ can be set as the mean or mode of $\gamma_{z}$ in the whole image. Using $\gamma_{s}=\gamma_{x} / b$, it can be obtained that $\gamma_{x}=\gamma_{s} b$. Through $\gamma_{z}=\gamma_{x} / a$ and $p=b / a<1, p$ can be calculated adaptively as a function of $\gamma_{z}$ that is estimated locally as $\gamma_{z} / \gamma_{s}$ and the texture becomes:

$$
t=\beta_{z}^{\gamma_{z} / \gamma_{s}} \Gamma\left(1 / \gamma_{s}\right) \mathrm{z}^{1-\left(\gamma_{z} / \gamma_{s}\right)} / \gamma_{s}
$$

If $\gamma_{z} \rightarrow \gamma_{S}$, there is a stark filtering in the image and if $\gamma_{z} \ll \gamma_{S}$ there is a weak filtering and, if $\gamma_{z}>\gamma_{S}$ the texture equation holds, but it is not Weibull-distributed anymore.

Figure 1. Shows the effect of $P$ parameter in an amplitude SAR C-band ENVISAT image. (a) The original image; (b) $P=0.2$, window $3 \times 3$; (c) $P=0.5$, window $3 \times 3$; (d) $P=0.8$, window $3 \times 3$.

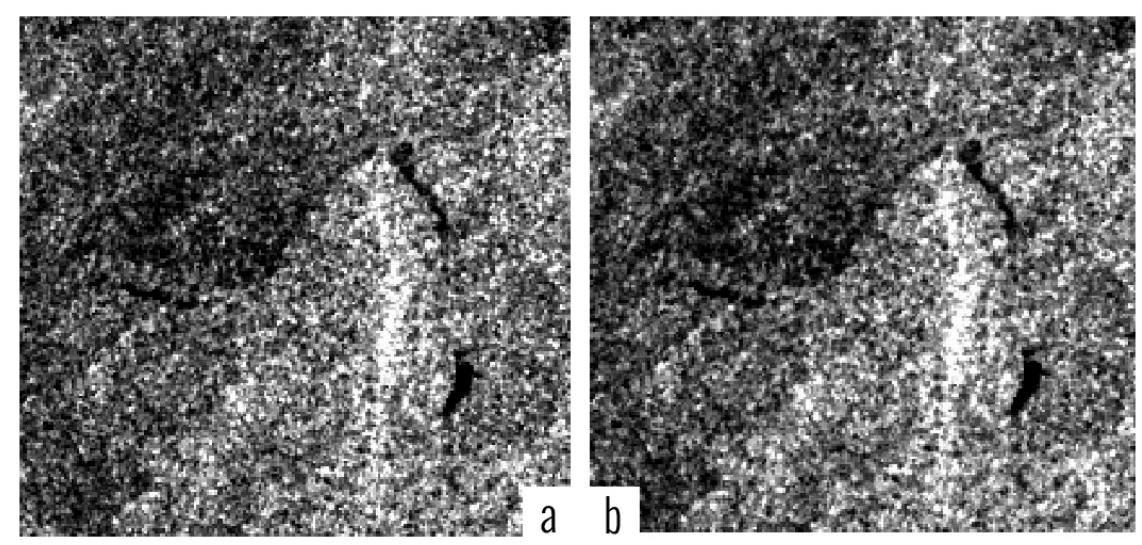


Figure 1. Cont.
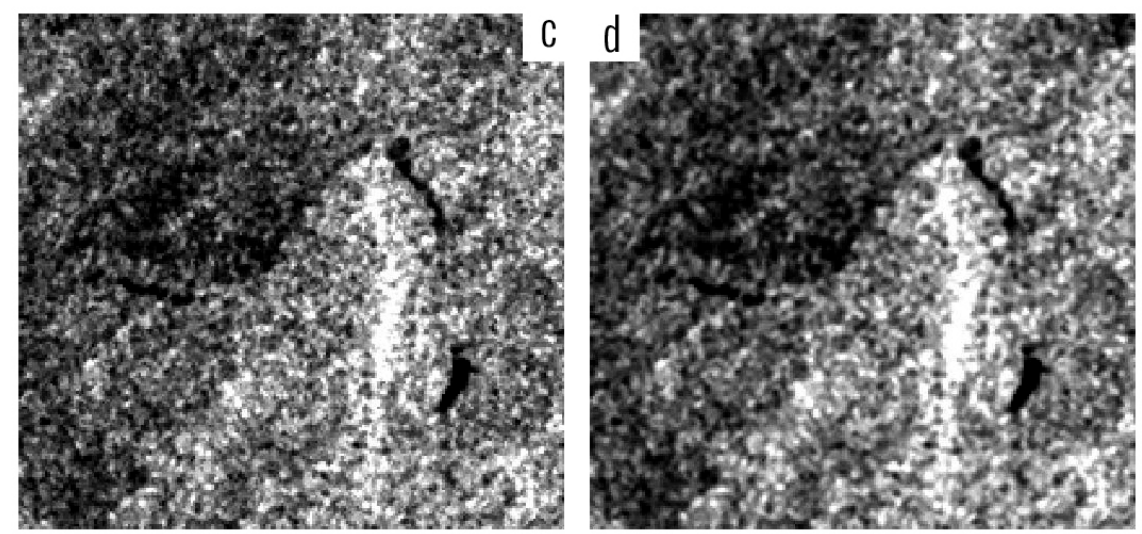

\subsection{MultiLayer Perceptron (MLP) Neural Networks}

The next step is segmenting the filtered image, in which the pixels are grouped according to the similarities by using MLP neural networks. A neuron k can be described by writing the following pair of equations [27]:

$$
\begin{gathered}
u_{k}=\sum_{i=1}^{n} w_{k i} x_{i} \\
y_{k}=\varphi\left(u_{k}+b_{k}\right)(x+a)^{n}=\sum_{k=0}^{n}\left(\begin{array}{l}
n \\
k
\end{array}\right) x^{k} a^{n-k}
\end{gathered}
$$

where $x_{1}, \ldots \ldots \ldots, x_{n}$ are the input signals, $w_{k 1}, \ldots \ldots \ldots, w_{k n}$ are the synaptic weights of neuron $\mathrm{k}, u_{k}$ is the linear combiner output due to the input signals, $b_{k}$ is the bias, $\varphi($.$) is the activation function, and$ $y_{k}$ is the output signal of the neuron. The logistic function is an example of a sigmoidal function which is the most commonly used activation function [1] is defined by the following equation where $a>0$ is the slope parameter:

$$
\varphi(v)=\frac{1}{1+e^{-a v}}
$$

The logistic function ranges from 0 to +1 ; however it is desirable to have the activation function range from -1 to +1 (anti-symmetric form). For the corresponding form of a sigmoid function we may use the hyperbolic tangent function, defined by:

$$
c(v)=\tanh (v)
$$

Allowing an activation function of the sigmoid type to assume negative values as prescribed by the above equation has analytic benefits [27].

Among neural network architectures, multilayer feedforward networks with MLPs as the learning algorithm are extensively used in pixel based oil spill segmentation [21,22]. Typically, MLPs consists of the input layer, one or more hidden layers of computation nodes, and an output layer of computation nodes. The input signal propagates through the network in a forward direction, on a layer-by-layer basis. 
Normalization is a preliminary phase of MLP segmentation which ensures that the distance measures respond with equal weight for each input [22]. Normalization performs by linear transformation from the image interval $[0,255]$ to the neural networks interval $[-1,1][22]$.

IDL programming language and the Stuttgart Neural Network Simulator (SNNS) developed at the University of Stuttgart, Germany [28], has been used for developing the WMM model and the classification algorithm implementation. The WMM filter model be obtained in IDL from website [29].

\section{Results and Discussion}

The model has been tested on a dataset of ENVISAT-ASAR (ASAR Image Mode Medium-resolution Image (IMM) and Wide Swath Mode (WSM) products which have a spatial resolution of $150 \mathrm{~m}$ ) and ERS2-SAR (PRecision Image (PRI) product which has a pixel size of $12.5 \mathrm{~m} \times 12.5 \mathrm{~m}$ ) images.

The dataset has been categorized into four groups which are massive well-defined, linear well-defined, massive not well-defined, linear not well-defined, (See [3]: Table 1) based on different types of dark spot and different sea status.

Table 1. The average values of the accuracies for different types of anomalies.

\begin{tabular}{ccccc}
\hline DARK Spot Types & Min\% & Max\% & Mean\% & StDev \\
\hline Well-Defined & 95.50 & 98.00 & 96.70 & 0.64 \\
Linear Well-Defined & 95.50 & 97.80 & 96.50 & 0.59 \\
Massive Well-Defined & 96.00 & 98.00 & 96.98 & 0.62 \\
Not Well-Defined & 87.00 & 94.00 & 92.55 & 1.81 \\
Linear Not Well-Defined & 87.00 & 94.00 & 92.97 & 2.00 \\
Massive Not Well-Defined & 87.50 & 93.10 & 92.13 & 1.58 \\
Linear Dark Spot & 87.00 & 97.80 & 94.74 & 2.31 \\
Massive Dark Spot & 87.50 & 98.00 & 94.55 & 2.74 \\
\hline
\end{tabular}

Radiometric calibration and geometric correction have been applied to the dataset in order to generate a backscatter $\left(\sigma^{0}\right)$ image, and to georeference the input images into the UTM projection with the WGS 84 as datum [3]. After calibration process, sub images containing all potential anomalies detected under a variety of sea conditions were extracted to make extraction more expedient. The test dataset contains 40 images with $256 \times 256$ pixels (around $4 \mathrm{~km}^{2}$ ), 20 images with $512 \times 512$ pixels (around $36 \mathrm{~km}^{2}$ ).

We applied adaptive WMM filter to all 60 test images. The similarity of adaptive and non-adaptive WMM filtered image has been shown in Figure 2. In the example shown in Figure 2, the filtering intensity $\mathrm{P}=0.7$ and a $3 \times 3$ window has been used for non-adaptive WMM (which was found to be the most appropriate combination of parameters for the WMN filter based on the previous studies) [3].

Removing the noisy pixels in the images by using adaptive WMM overcomes the non-adaptive WMM filter setting parameters while preserving the same accuracy of the non-adaptive model.

The classification task is based on the MLP approach. In designing the MLP model the number of units in the hidden layer and the training/testing phase settings (number of training cycles and the pixel selection for training/test the model) represent the fundamental tasks. Adjustment of these parameters affects the capability and sensitivity of the model to fit at the dynamic range of the backscattering values in the scene. 
Figure 2. Shows an example of adaptive (b) and non-adaptive (c) WMM filtering of the original image (a).

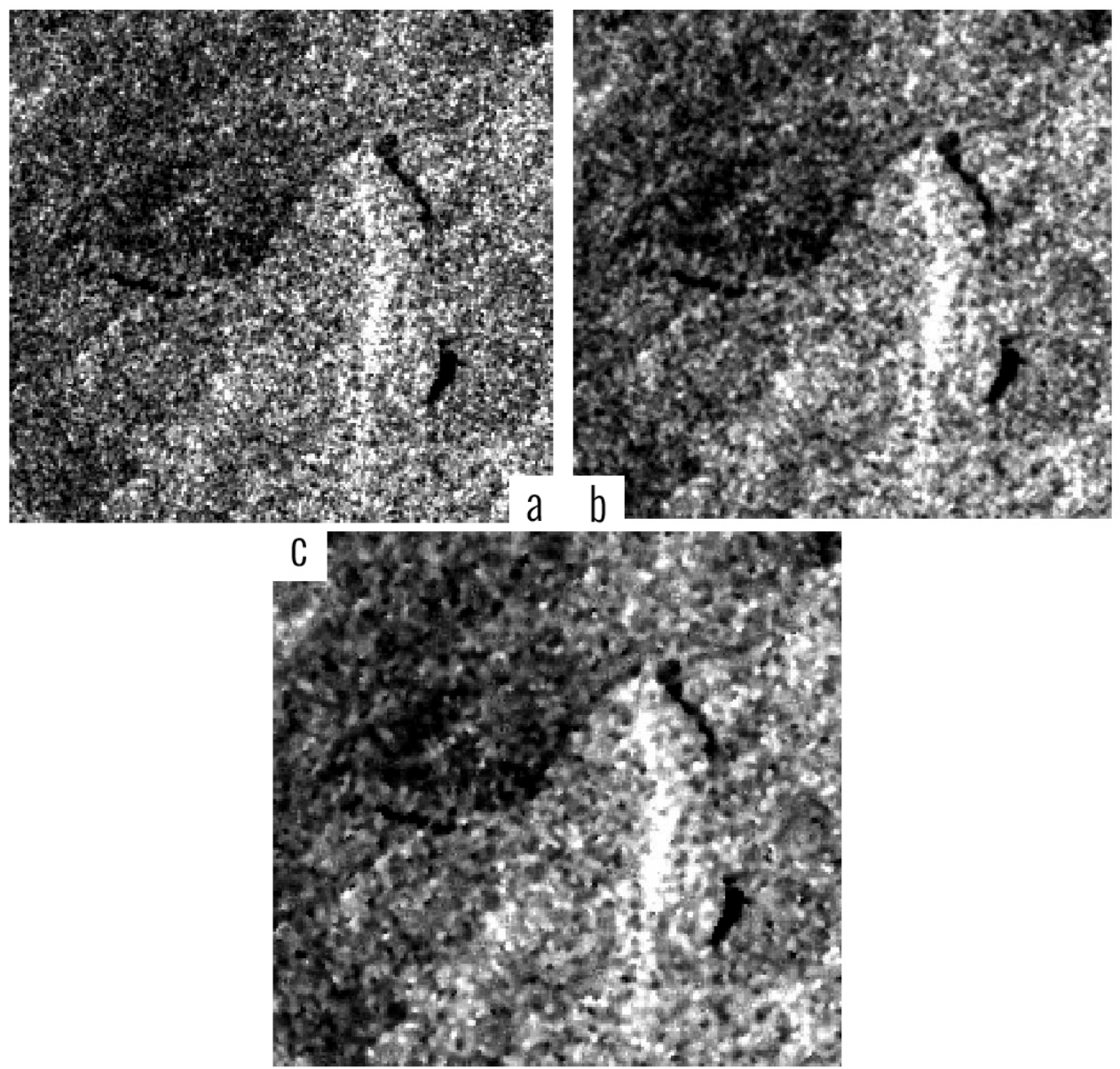

Several attempts have been made to properly select the number of units to be considered in the hidden layers. For training/test of the neural net 7000 pixels were extracted from different types of dark spots and different sea status. The tested windows were chosen to be as different as possible in order to test the neural networks ability to generalize different types of dark formations. The training and test sets were independent, the former containing $60 \%$ and the latter $40 \%$ of all pixels.

Pixel selection for training/test set has been done randomly and repeated six times. The presented results of root mean square error (RMSE) errors in Figure 3 are the average of these repetitions for each topology. The topology 1-4-2 has been finally chosen for its good performance in terms of classification accuracy, RMSE, and training time. A number of about 5000 training cycles was sufficient to get the network trained. The input of the net is the filtered image and the output providing classified pixels in terms of oil spill or others. However, after training the network for one specific sensor and product, no further tuning is necessary. After the segmentation phase, a very simple filtering process is used to eliminate all the objects with an area of less than 20 pixels from the processed image [3].

One MLP neural network (with the topology 1-4-2) has been used for classifying all images. In the next phase, for accuracy assessment, 500 pixels have randomly been selected from each sub-image and then labeled (oil spill or others) by visual interpretation. The results have been compared with the results of non-adaptive WMM \& PCNN model which is the most recent method in literature presented by Taravat et al. [3]. 
In some cases of the Wide Swath products non-adaptive WMM \& PCNN generates poor accuracies because the strong variation of incidence angle from near to far range affects the dynamic range in the images [3].

Figure 3. RMSE errors for different neural network topologies.

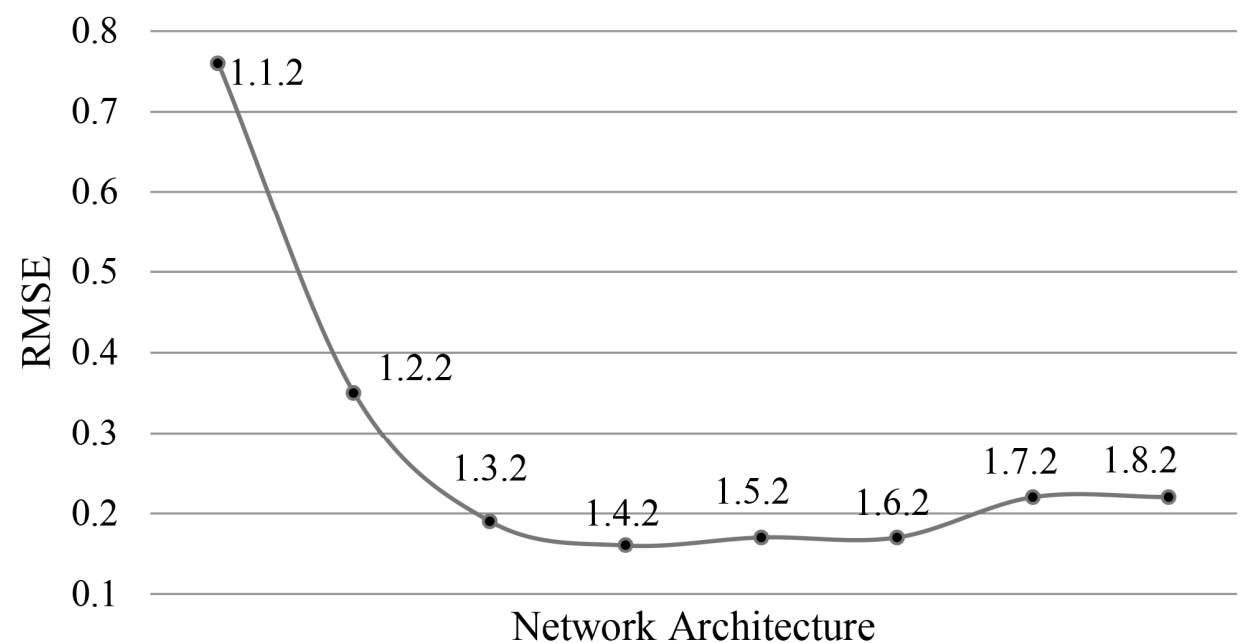

Figure 4 shows two sample test images from cases where non-adaptive WMM \& PCNN generates poor accuracies. The results of adaptive WMM filter and MLP segmentation are presented in the second and third row of Figure 4, respectively. The fourth row presents the final results after post processing.

Figure 4. Results of the proposed approach on two typical examples where non-adaptive WMM \& PCNN generates poor accuracies. (a) Original SAR images after preprocessing; (b) adaptive WMM filtering; (c) MLP results; (d) Final results after post processing.
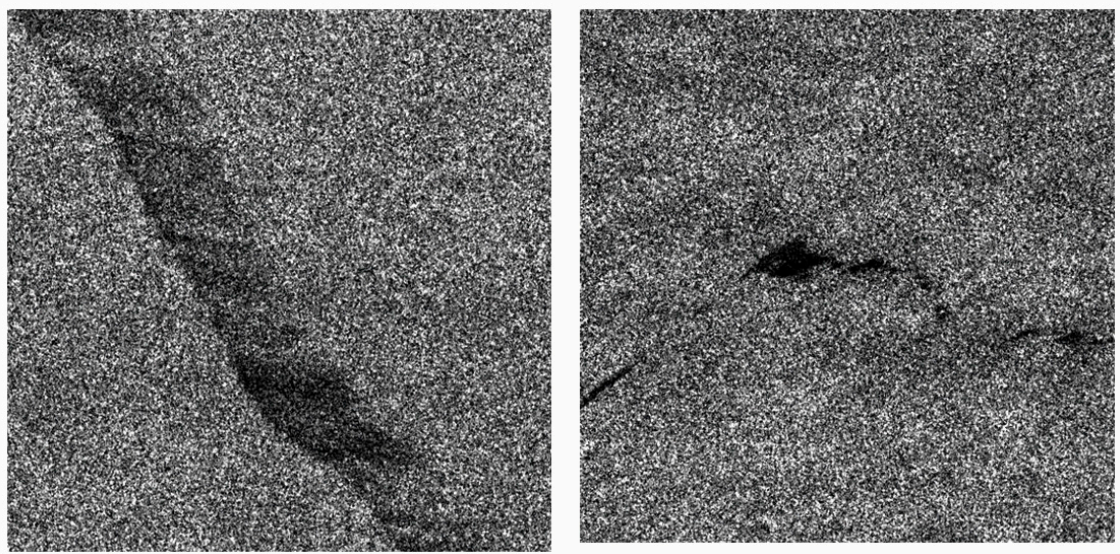

(a) 
Figure 4. Cont.
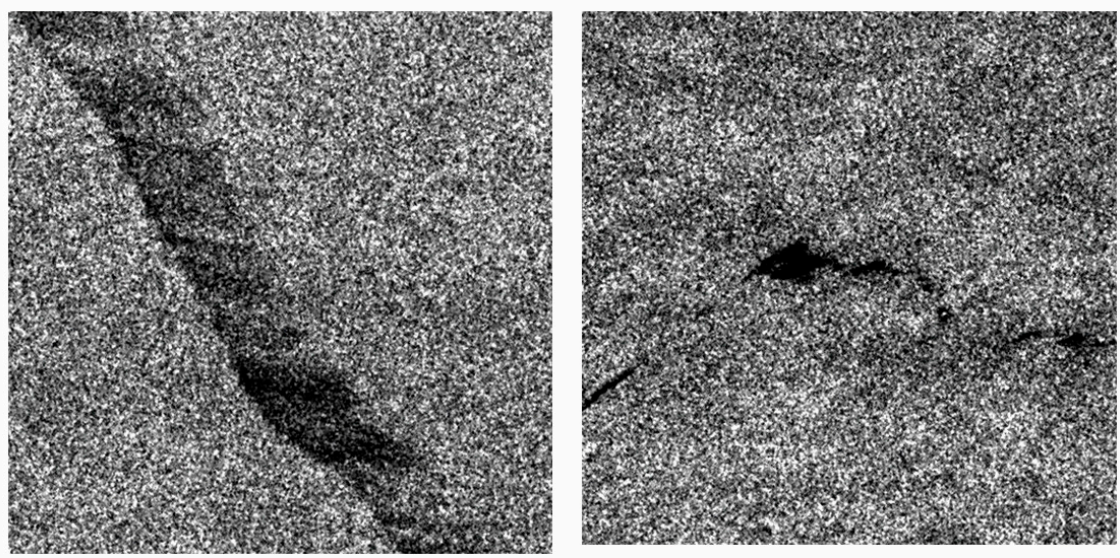

(b)

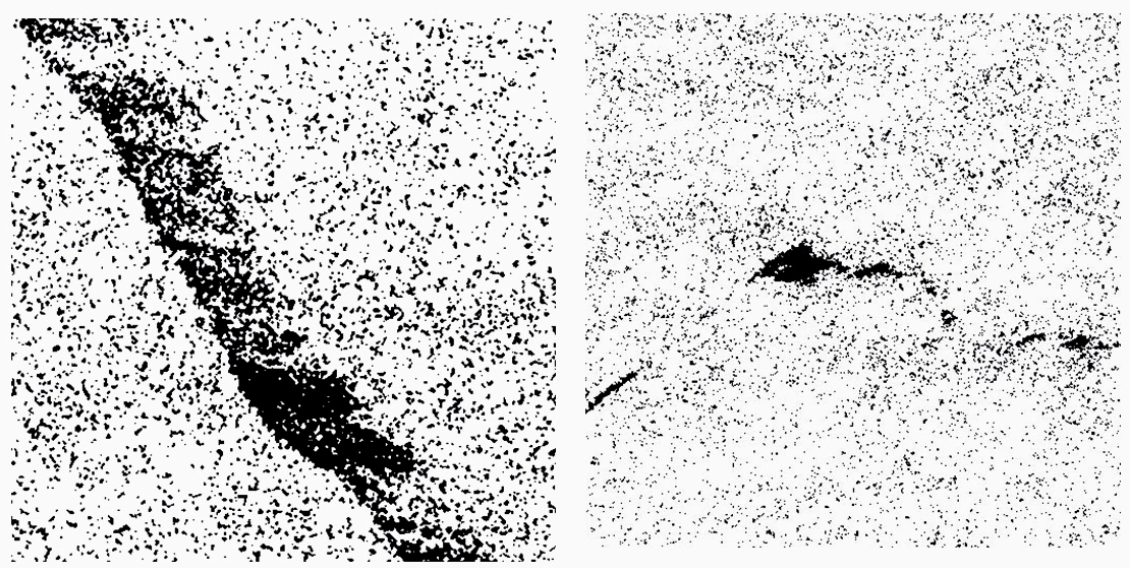

(c)

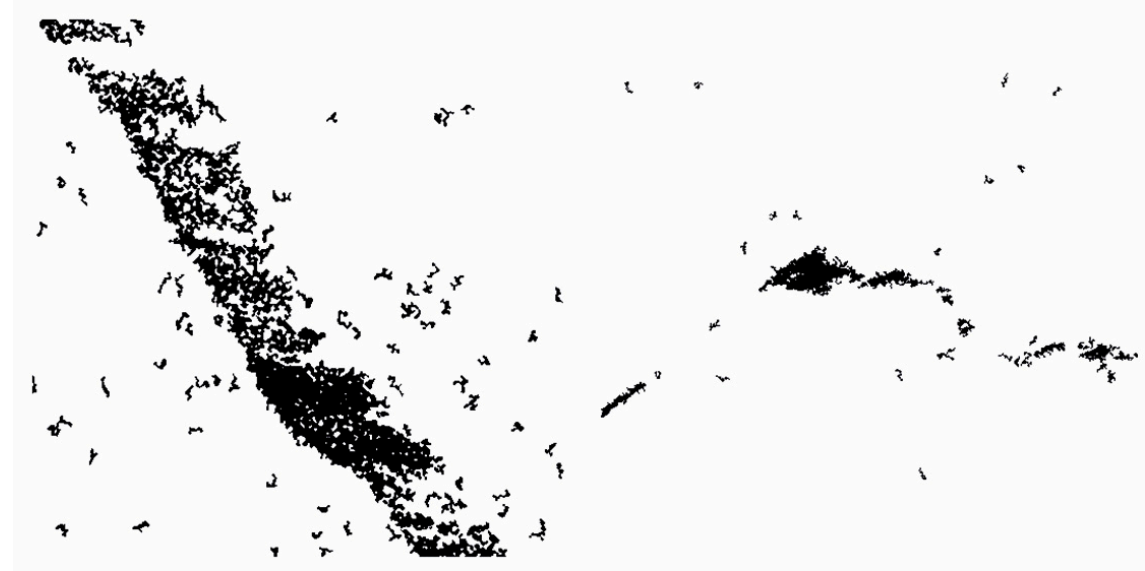

(d)

A not-well-defined massive dark spot and a not-well-defined linear dark spot are displayed in the right and left columns, respectively. Not-well-defined dark spots occur either when a fresh oil spill is present on a bright background or when the background is heterogeneous, resulting in a large number of false alarms after applying the model.

The accuracy of the entire test dataset increased with $1.1 \%$; moreover, standard deviation shows a significant improvement 1.3 (94.65\% with a standard deviation of 2.5$)$ in comparison to the same dataset segmented by non-adaptive WMM \& PCNN (93.53\% with a standard deviation of 3.8$)$. 
In the worst case, an accuracy of $87 \%$ was produced which is higher than the worst case accuracy presented in Taravat et al. (which is $84.88 \%$ ). The results of the accuracy assessment applied to the different types of anomalies are displayed in Tables 1 and 2.

The approach generates almost similar accuracies on well-defined dark spots in comparison to the accuracies of well-defined dark spots segmented by non-adaptive WMM \& PCNN. A significant improvement of $2.46 \%$ in accuracy (with the improvement of 0.44 in standard deviation and $2.4 \%$ in commission error) has been detected on the not well-defined dark spot dataset segmented by adaptive WMM \& MLP in comparison with the same dataset segmented by non-adaptive WMM \& PCNN (not well-defined linear dark spots accuracy is $90.36 \%$ with a standard deviation of 2.36 and commission error of $10.32 \%$. Not well-defined massive dark spots accuracy is $89.81 \%$ with a standard deviation of 2.22 and commission error of $11.70 \%$ ).

Table 2. The average values of emission and commission error (In \%) achieved by adaptive WMM \& MLP.

\begin{tabular}{lcccccccc}
\hline \multirow{2}{*}{ Dark Spot Types } & Min & Max & Mean & StDev & Min & Max & Mean & StDev \\
& Om & Om & Om & Om & Cm & Cm & Cm & Cm \\
\hline Well-Defined & 2.00 & 4.50 & 3.25 & 0.64 & 1.10 & 3.50 & 2.30 & 0.62 \\
Linear Well-Defined & 2.20 & 4.50 & 3.48 & 0.59 & 1.10 & 3.50 & 2.24 & 0.62 \\
Massive Well-Defined & 2.00 & 4.00 & 3.01 & 0.62 & 1.40 & 3.20 & 2.37 & 0.64 \\
Not Well-Defined & 6.00 & 13.0 & 7.44 & 1.81 & 6.20 & 11.4 & 8.60 & 1.36 \\
Linear Not Well-Defined & 6.00 & 13.0 & 7.00 & 2.00 & 6.20 & 10.3 & 8.22 & 1.04 \\
Massive Not Well-Defined & 6.90 & 12.5 & 7.86 & 1.58 & 6.50 & 11.4 & 8.97 & 1.58 \\
Linear Dark Spot & 2.20 & 13.0 & 5.20 & 2.31 & 1.10 & 10.3 & 5.23 & 3.17 \\
Massive Dark Spot & 2.00 & 12.5 & 5.44 & 2.74 & 1.40 & 11.4 & 5.67 & 3.57 \\
\hline
\end{tabular}

The worst accuracies are $87.00 \%$ with $10.3 \%$ commission error and $87.5 \%$ with $11.4 \%$ commission error which are obtained for not well-defined linear dark spots and not well-defined massive dark spots that are $2 \%$ with $3.3 \%$ commission error and $2.62 \%$ with $2.8 \%$ commission error higher than the worst accuracies obtained by non-adaptive WMM \& PCNN for not well-defined linear dark spots and not well-defined massive dark spots, respectively.

MLP Neural Networks (as a pixel based classification model) are less sensitive to noise and give good performance for spots with weak edges because they utilize the statistical information within or outside the training set and this is the reason of the higher accuracies obtained by adaptive WMM \& MLP for not well-defined linear dark spots and not well-defined massive dark spots in compared to the accuracies obtained by non-adaptive WMM \& PCNN for not well-defined linear dark spots and not well-defined massive dark spots.

\section{Conclusions}

In the present study a detailed research on the ability of using adaptive WMM \& MLP as an improved method of non-adaptive WMM \& PCNN model for dark-spot detection in SAR imagery is demonstrated. Adaptive WMM model presented in this study overcomes the non-adaptive WMM filter setting parameters. Furthermore a pixel-based classification model (MLP neural network) has been applied to a test dataset 
including 60 ENVISAT-ASAR and ERS2-SAR images. The same parameters were used for all the test images.

The whole test dataset accuracy is $94.65 \%$, which is higher than the same dataset segmented by non-adaptive WMM \& PCNN (93.53\%). The approach generates almost similar accuracy on well-defined dark spots in comparison to the accuracy on well-defined dark spots segmented by non-adaptive WMM \& PCNN. Results showed that this approach works better in the situations (not well-defined linear dark spots and not well-defined massive dark spots) where non-adaptive WMM \& PCNN generates poor accuracy.

Determination of appropriate characteristics for the training data and number of layers and nodes in the network topology are the main difficulties experienced in the use of many machine learning models, but once the topology and the other parameters are set, it can be used easily and very fast. The proposed approach can be applied well to the other spaceborn SAR (i.e., Sentinel-1) with some parameter adjustment based on the type of data.

\section{Acknowledgments}

The authors wish to thank David Fernandes, Head of Telecommunications Department, Centro Técnico de Aeronáutica (CTA) - Instituto Tecnológico de Aeronáutica (ITA), Brazil for their valuable cooperation and helpful suggestions. Data provided by European Space Agency (ESA) on the Cat-1 proposal No. 13926.

\section{Author Contributions}

Model development, and the experiment conceive, design and performs has been done by Alireza Taravat; the paper correction has been done by Natascha Oppelt.

\section{Conflicts of Interest}

The authors declare no conflict of interest.

\section{References}

1. Taravat, A.; Del Frate, F. Development of band ratioing algorithms and neural networks to detection of oil spills using landsat etm plus data. Eurasip J. Adv. Signal Process. 2012, 2012, 107.

2. Mera, D.; Cotos, J.M.; Varela-Pet, J.; Garcia-Pineda, O. Adaptive thresholding algorithm based on SAR images and wind data to segment oil spills along the northwest coast of the iberian peninsula. Mar. Pollut. Bull. 2012, 64, 2090-2096.

3. Taravat, A.; Latini, D.; Del Frate, F. Fully automatic dark-spot detection from SAR imagery with the combination of nonadaptive weibull multiplicative model and pulse-coupled neural networks. IEEE Trans. Geosci. Remote Sens. 2014, 52, 2427-2435.

4. Curlander, J.C.; McDonough, R.N. Synthetic Aperture Radar: Systems and Signal Processing; Wiley: New York, NY, USA, 2001.

5. Girard-Ardhuin, F.; Mercier, G.; Collard, F.; Garello, R. Operational oil-slick characterization by SAR imagery and synergistic data. IEEE J. Ocean. Eng. 2005, 30, 487-495. 
6. Jones, A.T.; Thankappan, M.; Logan, G.A.; Kennard, J.M.; Smith, C.J.; Williams, A.K.; Lawrence, G.M. Coral spawn and bathymetric slicks in synthetic aperture radar (SAR) data from the timor sea, north-west australia. Int. J. Remote Sens. 2006, 27, 2063-2069.

7. Thankappan, M.; Rollet, N.; Smith, C.J.H.; Jones, A.; Logan, G.; Kennard, J. Assessment of SAR ocean features using optical and marine survey data. In Proceedings of the Envisat Symposium, Montreux, Switzerland, 23-27 April 2007.

8. Ouchi, K. Recent trend and advance of synthetic aperture radar with selected topics. Remote Sens. 2013, 5, 716-807.

9. Marino, A.; Cloude, S.R.; Woodhouse, I.H. A polarimetric target detector using the huynen fork. IEEE Trans. Geosci. Remote Sens. 2010, 48, 2357-2366.

10. Margarit, G.; Milanes, J.A.B.; Tabasco, A. Operational ship monitoring system based on synthetic aperture radar processing. Remote Sens. 2009, 1, 375-392.

11. Brekke, C.; Solberg, A.H.S. Oil spill detection by satellite remote sensing. Remote Sens. Environ. 2005, 95, 1-13.

12. Nirchio, F.; Sorgente, M.; Giancaspro, A.; Biamino, W.; Parisato, E.; Ravera, R.; Trivero, P. Automatic detection of oil spills from SAR images. Int. J. Remote Sens. 2005, 26, 1157-1174.

13. Karathanassi, V.; Topouzelis, K.; Pavlakis, P.; Rokos, D. An object-oriented methodology to detect oil spills. Int. J. Remote Sens. 2006, 27, 5235-5251.

14. Keramitsoglou, I.; Cartalis, C.; Kiranoudis, C.T. Automatic identification of oil spills on satellite images. Environ. Model. Softw. 2006, 21, 640-652.

15. Solberg, A.H.S.; Brekke, C.; Husoy, P.O. Oil spill detection in radarsat and envisat SAR images. IEEE Trans. Geosci. Remote Sens. 2007, 45, 746-755.

16. Olsen, R.B.; Wahl, T. The role of wide swath SAR in high-latitude coastal management. Johns Hopkins APL Tech. Dig. 2000, 21, 136-140.

17. Palenzuela, J.M.T.; Vilas, L.G.; Cuadrado, M.S. Use of asar images to study the evolution of the prestige oil spill off the galician coast. Int. J. Remote Sens. 2006, 27, 1931-1950.

18. Li, Y.; Li, J. Oil spill detection from SAR intensity imagery using a marked point process. Remote Sens. Environ. 2010, 114, 1590-1601.

19. Shu, Y.M.; Li, J.; Yousif, H.; Gomes, G. Dark-spot detection from SAR intensity imagery with spatial density thresholding for oil-spill monitoring. Remote Sens. Environ. 2010, 114, 2026-2035.

20. Del Frate, F.; Petrocchi, A.; Lichtenegger, J.; Calabresi, G. Neural networks for oil spill detection using ers-SAR data. IEEE Trans. Geosci. Remote Sens. 2000, 38, 2282-2287.

21. Topouzelis, K.; Karathanassi, V.; Pavlakis, P.; Rokos, D. Detection and discrimination between oil spills and look-alike phenomena through neural networks. ISPRS J. Photogramm. 2007, 62, 264-270.

22. Topouzelis, K.; Karathanassi, V.; Pavlakis, P.; Rokos, D. Dark formation detection using neural networks. Int. J. Remote Sens. 2008, 29, 4705-4720.

23. Topouzelis, K.N. Oil spill detection by SAR images: Dark formation detection, feature extraction and classification algorithms. Sensors 2008, 8, 6642-6659.

24. Liu, P.; Zhao, C.F.; Li, X.F.; He, M.X.; Pichel, W. Identification of ocean oil spills in SAR imagery based on fuzzy logic algorithm. Int. J. Remote Sens. 2010, 31, 4819-4833.

25. Kuruoglu, E.E.; Zerubia, J. Modeling SAR images with a generalization of the rayleigh distribution. IEEE Trans. Image Process. 2004, 13, 527-533. 
26. Fernandes, D. Segmentation of SAR images with weibull distribution. In Proceedings of the 1998 IEEE International Geoscience and Remote Sensing Symposium, 1998 (IGARSS '98), Washington, DC, USA, 6-10 July 1998; Volume 21, pp. 24-26.

27. Bishop, C.M. Neural Networks for Pattern Recognition; Oxford University Press: Oxford, UK, 1995.

28. Zell, A.; Mamier, G.; Vogt, M.; Mache, N.; Ralf Hubner, S.D.; Herrmann, K.-U.; Soyez, T.; Schmalzl, M.; Sommer, T.; Hatzigeorgiou, A.; et al. Stuttgart Neural Network Simulator (SNNS), version 4.3; University of Stuttgart: Stuttgart, Germany, 1995.

29. Geoinformation Online. Available online: www.geoinformation.com (accessed on 1 December 2014).

(C) 2014 by the authors; licensee MDPI, Basel, Switzerland. This article is an open access article distributed under the terms and conditions of the Creative Commons Attribution license (http://creativecommons.org/licenses/by/4.0/). 